Gut and Liver, Vol. 12, No. 1, January 2018, pp. 46-50

\title{
Additive Effects of Rebamipide Plus Proton Pump Inhibitors on the Expression of Tight Junction Proteins in a Rat Model of Gastro-Esophageal Reflux Disease
}

Tae-Geun Gweon ${ }^{1}$, Jong-Hyung Park ${ }^{2}$, Byung-Wook Kim ${ }^{1}$, Yang Kyu Choi ${ }^{2}$, Joon Sung Kim ${ }^{1}$, Sung Min Park ${ }^{1}$, Chang Whan $\mathrm{Kim}^{3}$, Hyung-Gil Kim ${ }^{4}$, Jun-Won Chung, and Incheon and Western Kyonggi Gastrointestinal Study Group

${ }^{1}$ Division of Gastroenterology, Department of Internal Medicine, Incheon St. Mary's Hospital, The Catholic University of Korea College of Medicine, Incheon, ${ }^{2}$ Department of Laboratory Animal Medicine, Konkuk University College of Veterinary Medicine, Seoul, ${ }^{3}$ Division of Gastroenterology, Department of Internal Medicine, Bucheon St. Mary's Hospital, The Catholic University of Korea College of Medicine, Bucheon, ${ }^{4}$ Division of Gastroenterology, Department of Internal Medicine, Inha University College of Medicine, and ${ }^{5}$ Division of Gastroenterology, Department of Internal Medicine, Gachon University Gil Medical Center, Incheon, Korea

Background/Aims: The aim of this study was to investigate the effects of rebamipide on tight junction proteins in the esophageal mucosa in a rat model of gastroesophageal reflux disease (GERD). Methods: GERD was created in rats by tying the proximal stomach. The rats were divided into a control group, a proton pump inhibitor (PPI) group, and a PPI plus rebamipide (PPI+R) group. Pantoprazole $(5 \mathrm{mg} / \mathrm{kg})$ was administered intraperitoneally to the PPI and PPI+R groups. An additional dose of rebamipide (100 mg/kg) was administered orally to the PPI+R group. Mucosal erosions, epithelial thickness, and leukocyte infiltration into the esophageal mucosa were measured in isolated esophagi 14 days after the procedure. A Western blot analysis was conducted to measure the expression of claudin-1, -3 , and -4 . Results: The mean surface area of mucosal erosions, epithelial thickness, and leukocyte infiltration were lower in the PPI group and the $\mathrm{PPI}+\mathrm{R}$ group than in the control group. Western blot analysis revealed that the expression of claudin- 3 and -4 was significantly higher in the PPI+R group than in the control group. Conclusions: Rebamipide may exert an additive effect in combination with PPI to modify the tight junction proteins of the esophageal mucosa in a rat model of GERD. This treatment might be associated with the relief of GERD symptoms. (Gut Liver 2018;12:46-50)

Key Words: Gastroesophageal reflux; Rebamipide; Tight junction protein

\section{INTRODUCTION}

Gastroesophageal reflux disease (GERD) is a chronic and troublesome condition when gastric content reflux into the esophagus and causes symptoms. ${ }^{1}$ Tissue resistance is one of the defense mechanisms of the esophagus and prevents mucosal damage from the gastric refluxate. However, refluxed gastric acid might increase the permeability of the esophageal mucosa and result in dilation of intercellular space (DIC)., ${ }^{2,3}$ Subsequently, gastric acid may diffuse through the DIC and activate the acid-sensitive nociceptors in the esophageal wall., ${ }^{3,4}$ DIC has been reported to be associated with GERD symptom, ${ }^{4,5}$ and administration of proton pump inhibitors (PPIs) have been shown to recover DIC.

Rebamipide, a mucosal protective agent, has various effects favoring ulcer healing and prevention of gastric injury induced by nonsteroidal anti-inflammatory drugs and Helicobacter pylori infections. ${ }^{6}$ Additional therapeutic efficacy of rebamipide in conjunction with PPI has been reported in patients with GERD. ${ }^{7}$ However, the mechanism of the exact synergistic effect of rebamipide and PPI is yet to be understood.

Claudin is a tight junction protein in the epithelium and endothelium and exerts critical barrier function of the tight junction. Claudin is found in tissues of the kidney, gastrointestinal tract, and respiratory tract. ${ }^{8}$ Recent studies showed that altered locations of claudins are associated with GERD and decrease of claudin-3 has been demonstrated in rats with GERD. ${ }^{9,10}$ We

Correspondence to: Byung-Wook Kim

Division of Gastroenterology, Department of Internal Medicine, Incheon St. Mary's Hospital, The Catholic University of Korea College of Medicine, 56 Dongsu-ro, Bupyeong-gu, Incheon 21431, Korea

Tel: +82-32-280-5052, Fax: +82-32-280-5987, E-mail: gastro@catholic.ac.kr

Received on February 17, 2017. Revised on April 10, 2017. Accepted on April 28, 2017. Published online October 27, 2017

pISSN 1976-2283 eISSN 2005-1212 https://doi.org/10.5009/gnl17078

Tae-Geun Gweon and Jong-Hyung Park contributed equally to this work as first authors.

@ This is an Open Access article distributed under the terms of the Creative Commons Attribution Non-Commercial License (http://creativecommons.org/licenses/by-nc/4.0) which permits unrestricted non-commercial use, distribution, and reproduction in any medium, provided the original work is properly cited. 
hypothesized that administration of rebamipide might improve these tight junction proteins and consequently improve GERD symptoms. In this study, we tried to investigate the effect of rebamipide on tight junction proteins of the esophageal mucosa in a rat model with GERD.

\section{MATERIALS AND METHODS}

\section{Creation of GERD in rats}

Male Sprague-Dawley rats aged 7 weeks were purchased from commercial provider and used in this study. The rats were fasted for 18 hours before surgery and anesthetized with ether. After midline incision, the junction between corpus and forestomach was tied, and the duodenum near the pyloric ring was wrapped with 0-ring as in other studies. ${ }^{9,10}$ Diet was restricted except water, normal saline, and glucose for 24 hours after surgery. The rats were divided into three groups; control group, PPI group, and PPI plus rebamipide (PPI+R) group. In all three groups, 0.5\% carboxymethyl cellulose (CMC; Sigma Chemical, St. Louis, MO, USA) in normal saline solution was administered intragastrically through a feeding needle. Pantoprazole was administered at $5 \mathrm{mg} / \mathrm{kg} /$ day intraperitoneally in PPI group and in PPI+R group until sacrificed. In PPI+R group, rebamipide was administered at $100 \mathrm{mg} / \mathrm{kg} /$ day into the stomach through a feeding needle immediately after administration of intraperitoneal PPI until sacrificed. Body weight was checked daily and the rats were sacrificed 14 days after creating GERD. The whole esophagus along with the proximal part of stomach was isolated and observed for evaluation of erosive lesions. These lesions were marked with a pen and the gross area of whole mucosal defects was calculated. A part of tissue was cut longitudinally and embedded in paraffin for hematoxylin and eosin (H\&E) stain and another part was frozen by liquid nitrogen for further studies. Epithelial thickness of the esophagus, defined as depth of the mucosal epithelium, was measured and leukocytes infiltration into the mucosal layer was assessed in three random high power fields in H\&E stained tissue $(\times 200)$. All experimental procedures were approved by Institutional Animal Care and Use Committee at Incheon St. Mary's Hospital, The Catholic University of Korea (No. CIMH-2014-001).

\section{Immunohistochemistry for claudins}

After cutting the paraffin-embedded tissues, they were incubated with blocking agents containing 0.5\% Blocking Reagent (PerkinElmer Life Science, Waltham, MA, USA), Tris-HCl (0.1 M, $\mathrm{pH}$ 7.5), and $\mathrm{NaCl}(0.15 \mathrm{M})$ for 30 minutes at room temperature, followed by a mixture of rabbit anti-claudin-1 antibody (1:150; Santa Cruz Biotechnology Inc., Santa Cruz, CA, USA) or anticlaudin-3 antibody (1:100; Merck Millipore, Billerica, MA, USA) or mouse anti-claudin-4 antibody (1:400; Santa Cruz Biotechnology Inc.) overnight at $4^{\circ} \mathrm{C}$. The sections were incubated with a mixture of goat anti-mouse IgG antibody (claudin-1 and clau- din-3) or goat anti-rabbit IgG antibody (claudin-4) for 1 hour at room temperature. The stained sections were viewed with a microscope after administration of 4'-6'-diamidino-2-phenylindole (DAPI).

\section{Measurement of claudin expressions by Western blot as- say}

Claudins-1, -3, and -4 expressions were measured by Western blot assay. In brief, the detached esophageal mucosa was homogenized in iced lysate buffer containing Tris- $\mathrm{HCl}(50 \mathrm{mmol} /$ $\mathrm{L}, \mathrm{pH}$ 8.0), $\mathrm{NaCl}$ (150 mmol/L), ethylenediaminetetraacetic acid (EDTA; $1 \mathrm{mmol} / \mathrm{L})$, and Triton X-100 (1\%). Then they were centrifuged at $12,000 \times g$ for 5 minutes at $4^{\circ} \mathrm{C}$ and then the supernatants were collected for analyses. Protein concentration was measured with protein assay kit (Thermo Fisher Scientific, Seoul, Korea). The sediments were loaded on $8 \%$ polyacrylamide gel electrophoresis at same amount of measured protein and were transferred electrophoretically to polyvinylidene difluoride transfer membrane. Blocking was done with 5\% milk powder for 2 hours, followed by incubation at $4^{\circ} \mathrm{C}$ for 1 day with rabbit anti-claudin-1, -3, and -4 antibodies. After washing the membrane, the tissue was incubated with goat anti-rabbit IgG conjugated with horseradish peroxidase at room temperature for 1 hour. The incubated membrane was analyzed using ECL Western blotting detection system (GE Healthcare System, Little Chalfont, UK). The expression of claudin-1, -3, and -4 was measured in comparison with $\beta$-actin.

\section{Statistical analysis}

Daily body weight change was expressed as percent of weight compared with initial body weight which was measured before surgery. Continuous variables were expressed as mean and standard deviation and were compared using student t-test. The measured expressions of claudin-1, -3 , and -4 were compared using analysis of variance test. A p-value less than 0.05 was

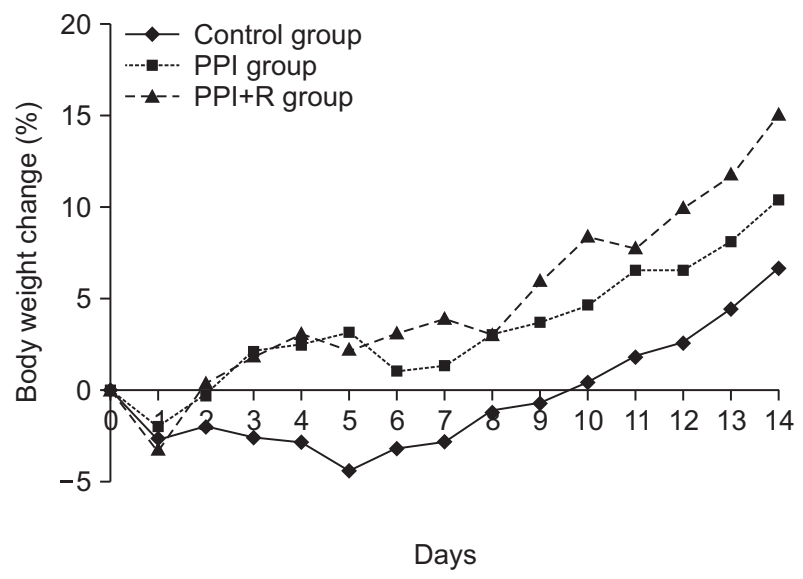

Fig. 1. Mean body weight changes after the creation of a rat reflux model. There were no differences among the groups. PPI, proton pump inhibitor; PPI+R, PPI plus rebamipide. 
considered statistically significant. Statistical analyses were conducted using SPSS version 18.0 (SPSS Inc., Chicago, IL, USA).

\section{RESULTS}

\section{Success of GERD model in rats}

One rat in the control group died after creating GERD due to over-sedation during the surgery. A total of 23 rats (control group, 7; PPI group, 8; PPI+R group, 8) completed this study. Mean body weight after fasting in control group, PPI group, and PPI+R group was 190.6, 195.5, and 194.9 g, respectively. Mean body weight at the end of study in each group was 203.5, 214.7, and $223.0 \mathrm{~g}$, respectively. Body weight change in each group is shown in Fig. 1. Body weight was increased in all groups at the end of study. PPI+R group showed the highest increase of body weight, but was not statistically significant.

\section{Macroscopic and microscopic findings}

Esophageal erosions were observed in 7 (100.0\%, 7/7), 2 $(25.0 \%, 2 / 8)$, and $3(37.5 \%, 3 / 8)$ rats in control group, PPI group, and PPI+R group, respectively. Mean surface area of the erosions in each group was $7.7 \pm 4.5,0.7 \pm 1.5$, and $0.7 \pm 1.3 \mathrm{~mm}^{2}$, respectively. The area of erosion in PPI group and in PPI $+R$ group was smaller than in control group ( $p=0.001, p=0.001$, respectively) (Fig. 2A). Epithelial thickness in each group was 118.6 \pm 35.2 , 81.0 \pm 17.8 , and $66.8 \pm 9.8 \mu \mathrm{m}$, respectively. Epithelial thickness in PPI group and in PPI+R group was significantly decreased than in control group ( $\mathrm{p}=0.047, \mathrm{p}=0.004$, respectively) (Fig. 2B). Number of infiltrated leukocytes in each group was $9.2 \pm 3.0,4.7 \pm 1.5,2.3 \pm 0.8$, respectively. Number of leukocyte
A

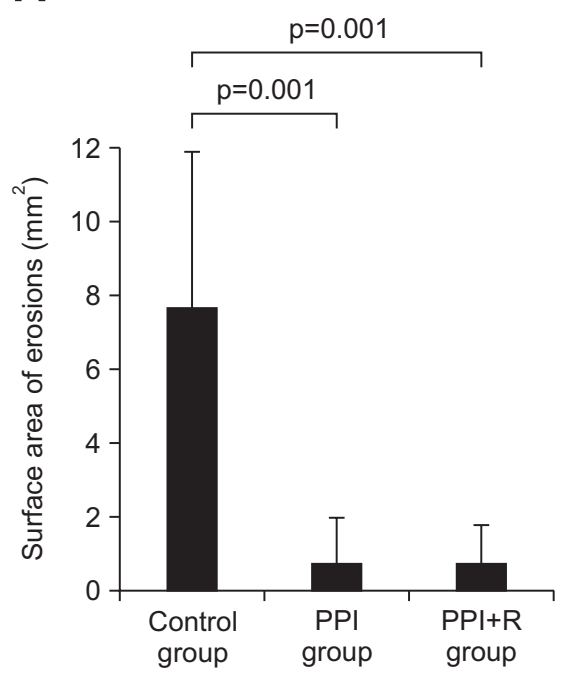

B

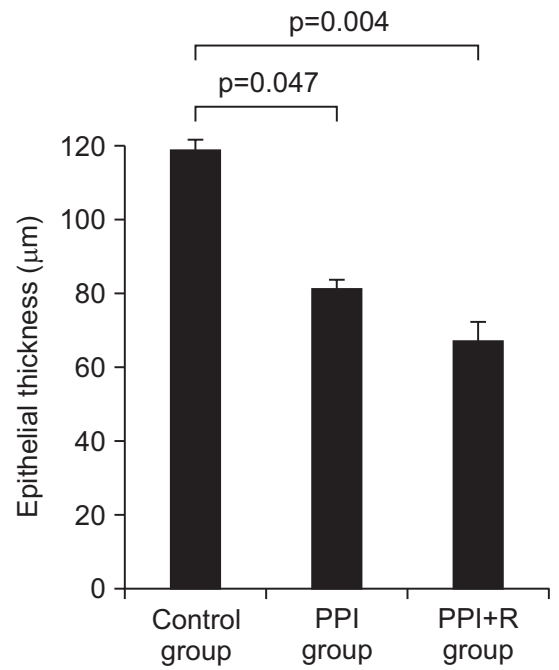

C

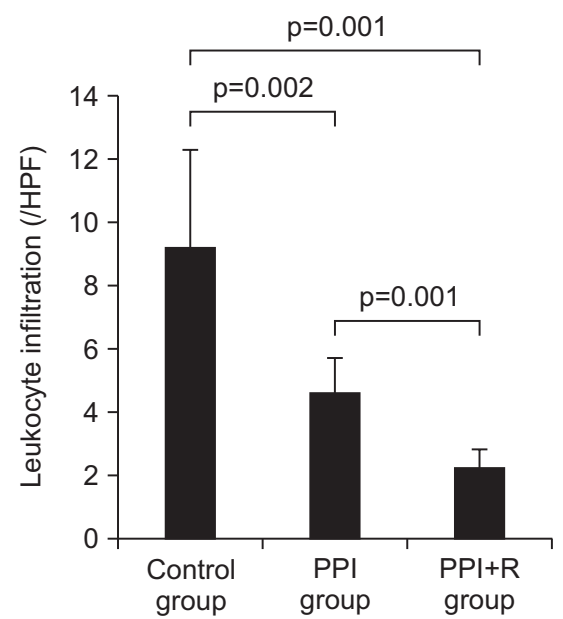

Fig. 2. (A) Surface area of erosions in each group. The control group showed larger erosion areas than the proton pump inhibitor (PPI) group and the PPI plus rebamipide (PPI+R) group. (B) Thickness of the esophageal epithelial layer in each group. The control group showed a thicker layer than the PPI group and the PPI+R group. (C) Number of leukocytes that infiltrated the lamina propria in each group. The number of infiltrating leukocytes was higher in the control group than in the PPI group and the PPI+R group, and the PPI group had a higher number of leukocytes than the PPI+R group.

HPF, high power field.

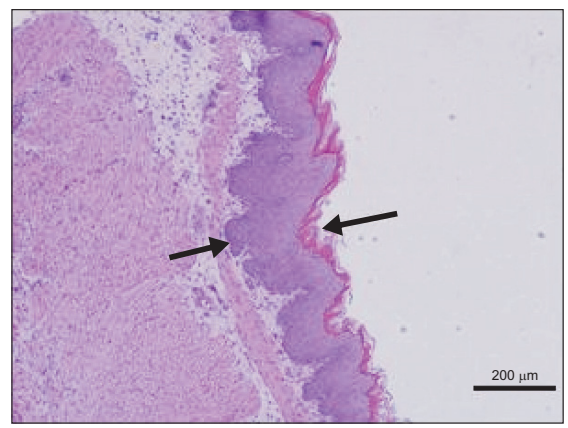

Control group

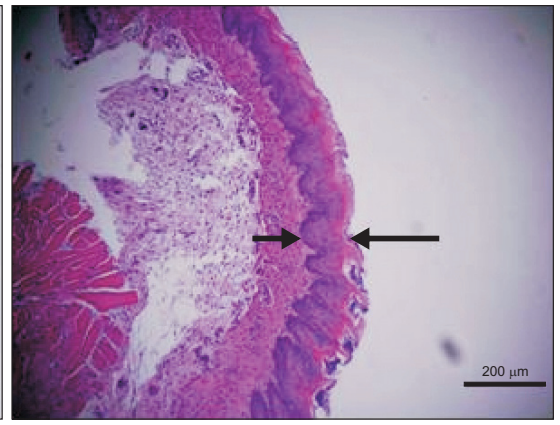

PPI group

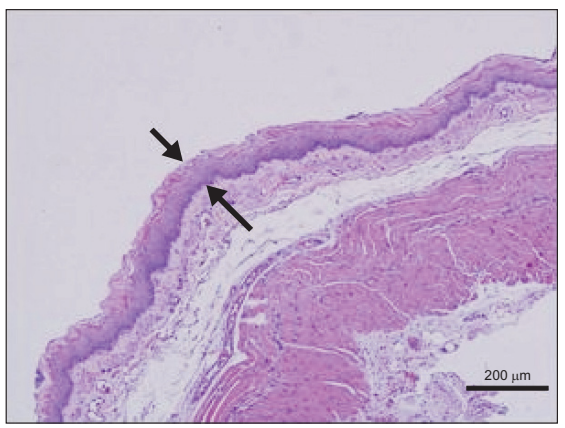

PPI+R group

Fig. 3. Hematoxylin and eosin staining of the esophageal mucosa in each group $(\times 100)$. Arrows indicate the thickness of the epithelial layer in each group. The control group had a thicker layer than the proton pump inhibitor (PPI) group and the PPI plus rebamipide (PPI+R) group. 
A

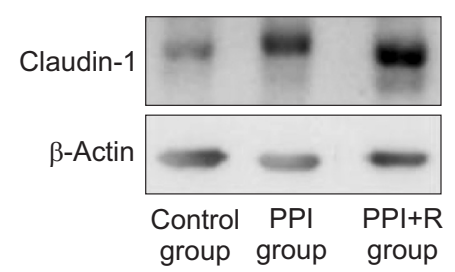

B

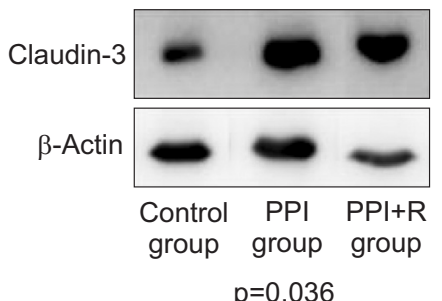

C

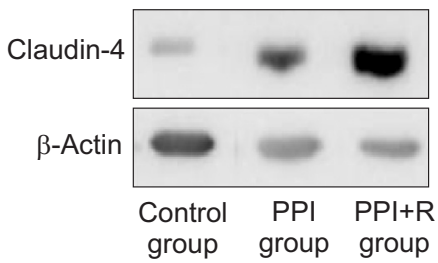

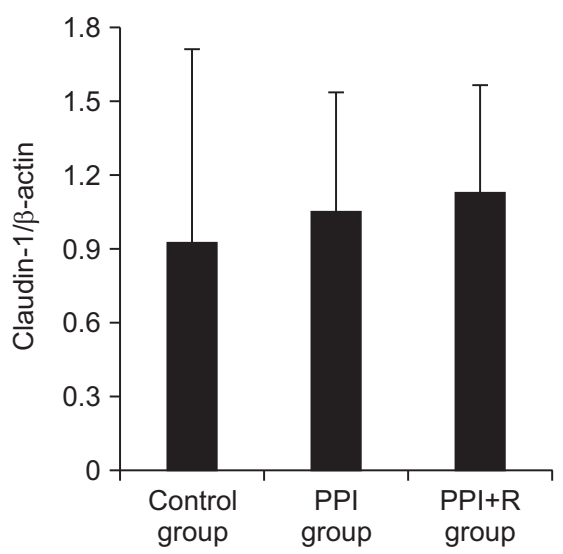
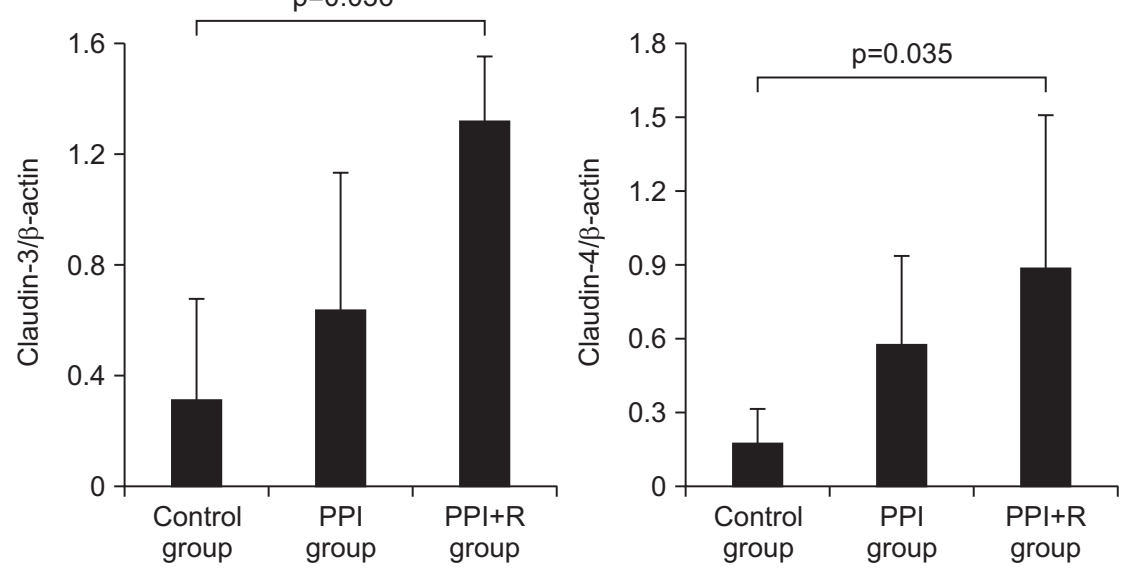

Fig. 4. (A) Western blot analysis for claudin-1 in each group. There were no differences among the groups. (B) Western blot analysis for claudin-3 in each group. The expression of claudin-3 decreased in the control group compared with the PPI+R group. (C) Western blot analysis for claudin-4 in each group. The expression of claudin- 4 decreased in the control group compared with the PPI+R group.

PPI, proton pump inhibitor; PPI+R, PPI plus rebamipide.

infiltration in PPI group and in PPI+R group was significantly lower than in control group ( $\mathrm{p}=0.002, \mathrm{p}=0.001$, respectively) (Fig. 2C). Fig. 3 shows the histology of each group.

\section{Immunohistochemical staining and Western blot of claudin}

On immunohistochemical staining, the expression of claudin-3 was decreased in control group compared with PPI group and in PPI+R group. On Western blot assay, the expression of claudin-1 in each group was $1.8 \pm 0.4,1.6 \pm 0.9$, and $1.6 \pm 0.8$, respectively. The expression of claudin-1 was not different in each group. The claudin-3 levels were measured as $0.6 \pm 0.0,1.2 \pm 0.2$, and $1.6 \pm 1.1$ in each group respectively (Fig. $4 \mathrm{~A}$ ). The expression of claudin-3 was significantly higher in PPI+R group compared with control group ( $p=0.036$ ) (Fig. 4B). The claudin -4 expression levels were measured as $0.2 \pm 0.1,0.6 \pm 0.3$, and $0.9 \pm 0.7$ in each group respectively. The expression of claudin-4 was significantly higher in PPI+R group compared with control group $(\mathrm{p}=0.035)$ (Fig. 4C).

\section{DISCUSSION}

Rebamipide has been shown to enhance the protective effect of esophageal mucosal in various animal models and in humans with GERD. ${ }^{7,11}$ However, the exact mechanism of this protective mechanism remained unknown. In the current study, we found that the administration of rebamipide along with PPI decreased the tight junction proteins of the esophageal mucosa. Rebamipide alone might also reduce the permeability of the mucosa in other parts of gastrointestinal tract, ${ }^{12,13}$ and work synergistically with PPIs in GERD patients. The expressions of claudin-3 and claudin-4 were only significantly higher in PPI+R group compared to control group. This suggests that PPI alone may not be sufficient in reducing the permeability of esophageal mucosa.

Decreased expression of claudin-3 and -4 was demonstrated in various GERD animal models and in humans with GERD. ${ }^{9,10,14,15}$ Another report showed that claudin-3 could be a sensitive indicator of reflux laryngitis in rats. ${ }^{16}$ Considering these reports together with our results, it is plausible that coadministration of rebamipide with PPI profoundly recovers the permeability of esophageal mucosa in rat GERD models.

Body weight of the rats increased in all groups after surgery in this study. Rats in PPI+R group showed the highest weight gain among all groups. Although it was not statistically significant, this finding implies the additive therapeutic efficacy of rebamipide over the single administration of PPI. Rebamipide might improve the reflux symptoms induced by reflux of gastric acid and hence food uptake was increased in our experimental models.

The number of mucosal erosions was significantly less in PPI group and in PPI+R group compared with control group. These results are in concordance with previous reports on the effect 
of enhanced ulcer healing in an animal model and iatrogenic gastric ulcer in humans. ${ }^{17,18}$ Our study showed that decreased thickness of epithelial layer and decreased number of leukocytes infiltrated in esophageal mucosa were more profound in PPI group and in PPI+R group compared with control group. From these findings we can conclude that PPI reduces inflammatory changes of the esophageal mucosa in GERD model and that rebamipide has additive effect. Our results support the previous report which showed that rebamipide inhibited the adherence of neutrophils to endothelial cell. ${ }^{19}$

There are several limitations to our study. There are differences between naturally occurring GERD and iatrogenic GERD pathophysiologically. Large amount of rebamipide directly contacts esophagus due to delayed gastric emptying in animal model while only small amount of rebamipide contacts esophagus in human GERD without obstruction.

In conclusion, administration of rebamipide along with PPI modifies tight junction proteins of esophageal mucosa, which resulted in prevention of GERD in a rat model. Further studies are anticipated to validate the effect of rebamipide in human esophageal mucosa.

\section{CONFLICTS OF INTEREST}

This study was granted from Otsuka Pharmaceutical Company (5-2013-D0248-00001).

\section{REFERENCES}

1. Vakil N, van Zanten SV, Kahrilas P, Dent J, Jones R; Global Consensus Group. The Montreal definition and classification of gastroesophageal reflux disease: a global evidence-based consensus. Am J Gastroenterol 2006;101:1900-1920.

2. Farré R, van Malenstein H, De Vos R, et al. Short exposure of oesophageal mucosa to bile acids, both in acidic and weakly acidic conditions, can impair mucosal integrity and provoke dilated intercellular spaces. Gut 2008;57:1366-1374.

3. van Malenstein H, Farré R, Sifrim D. Esophageal dilated intercellular spaces (DIS) and nonerosive reflux disease. Am J Gastroenterol 2008;103:1021-1028.

4. Caviglia R, Ribolsi M, Maggiano N, et al. Dilated intercellular spaces of esophageal epithelium in nonerosive reflux disease patients with physiological esophageal acid exposure. Am J Gastroenterol 2005;100:543-548.

5. Tobey NA, Carson JL, Alkiek RA, Orlando RC. Dilated intercellular spaces: a morphological feature of acid reflux--damaged human esophageal epithelium. Gastroenterology 1996;111:1200-1205.

6. Genta RM. Review article: the role of rebamipide in the management of inflammatory disease of the gastrointestinal tract. Aliment Pharmacol Ther 2003;18 Suppl 1:8-13.
7. Yoshida N, Kamada K, Tomatsuri N, et al. Management of recurrence of symptoms of gastroesophageal reflux disease: synergistic effect of rebamipide with $15 \mathrm{mg}$ lansoprazole. Dig Dis Sci 2010;55:3393-3398.

8. Günzel D, Yu AS. Claudins and the modulation of tight junction permeability. Physiol Rev 2013;93:525-569.

9. Asaoka D, Miwa H, Hirai S, et al. Altered localization and expression of tight-junction proteins in a rat model with chronic acid reflux esophagitis. J Gastroenterol 2005;40:781-790.

10. Oguro M, Koike M, Ueno T, et al. Dissociation and dispersion of claudin-3 from the tight junction could be one of the most sensitive indicators of reflux esophagitis in a rat model of the disease. J Gastroenterol 2011;46:629-638.

11. Katada K, Yoshida N, Isozaki Y, et al. Prevention by rebamipide of acute reflux esophagitis in rats. Dig Dis Sci 2005;50 Suppl 1:S97S103.

12. Matysiak-Budnik T, de Mascarel A, Abely M, Mayo K, Heyman M, Mégraud F. Positive effect of rebamipide on gastric permeability in mice after eradication of Helicobacter felis. Scand J Gastroenterol 2000;35:470-475.

13. Matysiak-Budnik T, Thomas-Collignon A, Mégraud F, Heyman M. Alterations of epithelial permeability by Helicobacter and IL-1beta in vitro: protective effect of rebamipide. Dig Dis Sci 2001;46:15581566.

14. Miwa H, Koseki J, Oshima T, et al. Rikkunshito, a traditional Japanese medicine, may relieve abdominal symptoms in rats with experimental esophagitis by improving the barrier function of epithelial cells in esophageal mucosa. J Gastroenterol 2010;45:478487.

15. Björkman EV, Edebo A, Oltean M, Casselbrant A. Esophageal barrier function and tight junction expression in healthy subjects and patients with gastroesophageal reflux disease: functionality of esophageal mucosa exposed to bile salt and trypsin in vitro. Scand J Gastroenterol 2013;48:1118-1126.

16. Xu XB, Hu Y, Wang Y, Lai CJ, Zhang TY. Expression of claudin-3 in the esophagus and larynx of rat reflux model. Auris Nasus Larynx 2014;41:539-542.

17. Arakawa T, Watanabe T, Fukuda T, Yamasaki K, Kobayashi K. Rebamipide, novel prostaglandin-inducer accelerates healing and reduces relapse of acetic acid-induced rat gastric ulcer. Comparison with cimetidine. Dig Dis Sci 1995;40:2469-2472.

18. Shin WG, Kim SJ, Choi MH, et al. Can rebamipide and proton pump inhibitor combination therapy promote the healing of endoscopic submucosal dissection-induced ulcers? A randomized, prospective, multicenter study. Gastrointest Endosc 2012;75:739747.

19. Yoshida N, Yoshikawa T, Iinuma S, et al. Rebamipide protects against activation of neutrophils by Helicobacter pylori. Dig Dis Sci 1996;41:1139-1144. 